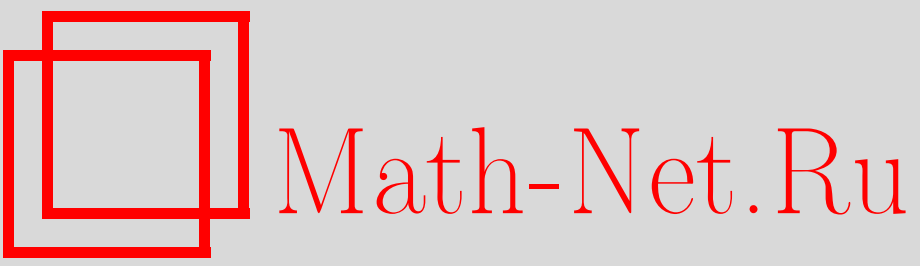

А. И. Назаров, Об одном семействе преобразований гауссовских случайных функций, Теория вероятн. и ее примен., 2009, том 54, выпуск 2, 209-225

DOI: https://doi.org/10.4213/tvp2696

Использование Общероссийского математического портала Math-Net.Ru подразумевает, что вы прочитали и согласны с пользовательским соглашением

http://www.mathnet.ru/rus/agreement

Параметры загрузки:

IP : 54.147 .182 .235

26 апреля 2023 г., 11:57:08 


\section{ОБ ОДНОМ СЕМЕЙСТВЕ ПРЕОБРАЗОВАНИЙ ГАУССОВСКИХ СЛУЧАЙНЫХ ФУНКЦИЙ ${ }^{1)}$}

В недавней статье [6] П. Деовельс показал, что для стандартного броуновского моста $B(t), 0 \leqslant t \leqslant 1$, справедливо равенство по распределению

$$
\mathscr{Y}_{K}(t) \stackrel{d}{=} \mathscr{Y}_{2-K}(t), \quad t \in[0,1], \quad K \in \mathbf{R},
$$

где $\mathscr{Y}_{K}(t)=B(t)-6 K t(1-t) \int_{0}^{1} B(s) d s$. Кроме того, им было получено явное разложение Карунена-Лоэва (КЛ) для процесса $\mathscr{Y}_{1}(t)$.

В настоящей работе для гауссовских случайных функций общего вида, имеющих нулевые средние, вводятся однопараметрические семейства преобразований, для которых выполнено соотношение, обобщающее $(*)$. В случае, когда $L_{2}$-норма исходной функции конечна п.н., выводится явное соотношение между точными асимптотиками вероятностей $L_{2}$-малых уклонений преобразованной и исходной функции. Для одномерных процессов, порождающих краевые задачи для обыкновенных дифференциальных уравнений, в случае, когда известно КЛ-разложение для исходного процесса, получено также КЛ-разложение для преобразованных процессов.

Ключевые слова и фразы: гауссовские случайные функции, разложение Карунена-Лоэва, определители Фредгольма, асимптотика вероятностей малых уклонений.

1. Основная лемма. Рассмотрим гауссовскую случайную функцию $X$ с нулевым средним. Для простоты предположим, что $X$ определена в замыкании $\overline{\mathscr{O}}$ ограниченной области $\mathscr{O} \subset \mathbf{R}^{n}$. Обозначим $G_{X}(x, y)=\mathbf{E} X(x) X(y)$ ковариационную функцию $X$.

Пусть $\varphi$ - локально суммируемая в $\mathscr{O}$ функция. Предположим, что функция

$$
\psi(x)=\int_{\mathscr{O}} G_{X}(x, y) \varphi(y) d y
$$

\footnotetext{
${ }^{*}$ Санкт-Петербургский государственный университет, математико-механический факультет, Университетский пр-т, 28, Старый Петергоф, 198504 С.-Петербург, Россия; e-mail: al.il.nazarov@gmail.com

1) Работа выполнена при поддержке программы «Ведущие научные школы» (грант НШ.227.2008.1) и РФФИ (грант № 07-01-00159).
} 
определена п.в. в $\mathscr{O}, \psi \neq \equiv 0$ и

$$
q=\int_{\mathscr{O}} \psi(u) \varphi(u) d u=\int_{\mathscr{O}} \int_{\mathscr{O}} G_{X}(u, v) \varphi(u) \varphi(v) d u d v<\infty .
$$

Построим семейство гауссовских функций $(\alpha \in \mathbf{R})$

$$
\mathscr{X}_{\varphi, \alpha}(x)=X(x)-\alpha \psi(x) \int_{\mathscr{O}} X(u) \varphi(u) d u, \quad x \in \overline{\mathscr{O}} .
$$

Основная лемма. Функиия $\mathscr{X}_{\varphi, \alpha}$ имеет ковариацию

$$
\mathscr{G}_{\varphi, \alpha}(x, y)=G_{X}(x, y)+Q \psi(x) \psi(y)
$$

где $Q=q \alpha^{2}-2 \alpha$.

Д о к а з а т е л ь с т в о. Проверяется непосредственным вычислением с учетом (1.1).

Следствие 1. Для процессов (1.3) имеет место равенство

$$
\mathscr{X}_{\varphi, \alpha}(x) \stackrel{d}{=} \mathscr{X}_{\varphi, 2 / q-\alpha}(x), \quad x \in \overline{\mathscr{O}} .
$$

В частности, $\mathscr{X}_{\varphi, 2 / q}(x) \stackrel{d}{=} X(x)$.

Следствие 2. Пусть $\widehat{\alpha}=1 / q$. Тогда:

1) имеет место тождество п.н.

$$
\int_{\mathscr{O}} \mathscr{X}_{\varphi, \hat{\alpha}}(x) \varphi(x) d x=0
$$

2) прочесс $\mathscr{X}_{\varphi, \hat{\alpha}}(x)$ и случайная величина $\int_{\mathscr{O}} X(u) \varphi(u) d u$ независимы;

3) если $\varphi \in L_{2}(\mathscr{O})$, то интегральный оператор с ядром $\mathscr{G}_{\varphi, \hat{\alpha}}(x, y)$ имеет нулевое собственное число, соответствующее собственной функции $\varphi$.

Д ок аз а тель с тв о. Все три утверждения следуют из несложно проверяемых соотношений

$$
\begin{aligned}
\int_{\mathscr{O}} \mathscr{X}_{\varphi, \alpha}(x) \varphi(x) d x & =\int_{\mathscr{O}} X(u) \varphi(u) d u \cdot(1-q \alpha) \\
\mathbf{E} \mathscr{X}_{\varphi, \alpha}(x) \int_{\mathscr{O}} X(u) \varphi(u) d u & =\psi(x)(1-q \alpha) .
\end{aligned}
$$

3 а м е ч а н и е. Очевидно, что $\mathscr{Y}_{K}$ совпадает с $\mathscr{B}_{\varphi, \alpha}$ при $\varphi \equiv 1$, $\alpha=12 K$. Поэтому лемма 2.2 и следствия 2.1 и $2.2[6]$ являются частными случаями наших утверждений. 
2. Асимптотика вероятности малых уклонений в $L_{2}$. Предположим теперь, что

$$
\|X\|_{2}^{2} \equiv \int_{\mathscr{O}} X^{2}(x) d x<\infty \quad \text { п.н. }
$$

Тогда для процесса $X$ справедливо разложение Карунена-Лоэва (КЛразложение)

$$
X(x) \stackrel{d}{=} \sum_{k=1}^{\infty} \sqrt{\lambda_{k}} u_{k}(x) \xi_{k}, \quad x \in \mathscr{O}
$$

где $\xi_{k}$ - последовательность независимых стандартных нормальных случайных величин, $\lambda_{k}>0$ и $u_{k}$ - соответственно собственные числа и нормированные в $L_{2}(\mathscr{O})$ собственные функции интегрального оператора $\mathfrak{G}$ с ядром $G_{X}(x, y)$. При этом $\sum_{k} \lambda_{k}<\infty$, т.е. оператор $\mathfrak{G}$ принадлежит ядерному классу $\mathfrak{S}_{1}$. Отметим, что ряд $(2.2)$ сходится в $L_{2}(\mathscr{O})$ п.н.

Из (2.2) следует, что

$$
\|X\|_{2}^{2} \stackrel{d}{=} \sum_{k=1}^{\infty} \lambda_{k} \xi_{k}^{2}
$$

Поэтому, зная собственные числа $\lambda_{k}$, можно получить информацию о распределении $\|X\|_{2}^{2}$ и, в частности, найти точную асимптотику вероятности малых уклонений в $L_{2}$, т.е. описать поведение при $\varepsilon \rightarrow 0$ вероятности $\mathbf{P}\left\{\|X\|_{2} \leqslant \varepsilon\right\}$.

В абстрактном виде решение задачи об асимптотике вероятности малых уклонений было получено в [22]. Затем многие авторы занимались упрощением выражения для вероятности малых уклонений при различных предположениях (см. статью [7] и имеющиеся в ней ссылки).

В статьях [18], [16], [17] был разработан подход, позволяющий получать асимптотику малых уклонений в $L_{2}$-норме для гауссовского процесса с нулевым средним, ковариация которого является функцией Грина для обыкновенного дифференциального оператора. В более общей ситуации решить задачу полностью пока не удается. Однако в рассматриваемом случае, поскольку оператор с ядром $\mathscr{G}_{\varphi, \alpha}(x, y)$ является одномерным возмущением исходного оператора, можно выразить асимптотику малых уклонений для $\mathscr{X}_{\varphi, \alpha}$ через асимптотику малых уклонений для исходного процесса.

3 а м е ч а н и е. Если оператор $\mathfrak{G}$ имеет нетривиальное нульпространство $U_{0}$, то в силу очевидного соотношения $\psi \perp U_{0}$ оно содержится в нуль-пространстве оператора с ядром $\mathscr{G}_{\varphi, \alpha}(x, y)$. Поэтому можно проводить все рассуждения в ортогональном дополнении к $U_{0}$ и считать, что в разложении (2.2) функции $\left\{u_{k}\right\}$ образуют полную ортонормированную систему. 
Теорема 1. Пусть выполнено условие (2.1) и функиия $\varphi \in$ $L_{1, \mathrm{loc}}(\mathscr{O})$ удовлетворяет условию (1.2). Если $\alpha \neq 1 / q$, то при $\varepsilon \rightarrow 0$

$$
\mathbf{P}\left\{\left\|\mathscr{X}_{\varphi, \alpha}\right\|_{2} \leqslant \varepsilon\right\} \sim \frac{1}{|1-\alpha q|} \mathbf{P}\left\{\|X\|_{2} \leqslant \varepsilon\right\} .
$$

Д о к а з а т е л ь с т в о. По теореме сравнения ([13]; см. также [8])

$$
\mathbf{P}\left\{\left\|\mathscr{X}_{\varphi, \alpha}\right\|_{2} \leqslant \varepsilon\right\} \sim \mathbf{P}\left\{\|X\|_{2} \leqslant \varepsilon\right\}\left(\prod_{k=1}^{\infty} \frac{\lambda_{k}}{\widetilde{\lambda}_{k}}\right)^{1 / 2},
$$

где $\widetilde{\lambda}_{k}$ - собственные числа интегрального оператора с ядром $\mathscr{G}_{\varphi, \alpha}(x, y)$. Отметим, что в силу минимаксимального принципа (см., например, $[4$, $\S 10.2])$ последовательности $\lambda_{k}$ и $\widetilde{\lambda}_{k}$ перемежаются. Отсюда, в частности, следует, что ряд $\sum_{k} \widetilde{\lambda}_{k}$ также сходится.

Обозначим $\mu_{k}=\lambda_{k}^{-1}, \widetilde{\mu}_{k}=\widetilde{\lambda}_{k}^{-1}$ и рассмотрим определители Фредгольма для ядер $G_{X}$ и $\mathscr{G}_{\varphi, \alpha}$ :

$$
\mathscr{F}(z)=\prod_{k=1}^{\infty}\left(1-\frac{z}{\mu_{k}}\right) ; \quad \widetilde{\mathscr{F}}(z)=\prod_{k=1}^{\infty}\left(1-\frac{z}{\widetilde{\mu}_{k}}\right) .
$$

В силу сходимости рядов $\sum_{k} \mu_{k}^{-1}$ и $\sum_{k} \widetilde{\mu}_{k}^{-1}$ эти канонические произведения Адамара сходятся при всех $z \in \mathbf{C}$. Ввиду (1.4) они связаны между собой формулой ${ }^{2}$

$$
\widetilde{\mathscr{F}}(z)=\mathscr{F}(z)\left(1+Q \sum_{k=1}^{\infty} \frac{a_{k}^{2} \mu_{k}}{1-\mu_{k} / z}\right),
$$

где $a_{k}$ - коэффициенты Фурье функции $\psi$ по полной ортонормированной системе $\left\{u_{k}\right\}$.

Из теоремы Йенсена (см. [23, §3.6]) следует, что

$$
\prod_{k=1}^{\infty} \frac{\mu_{k}}{\widetilde{\mu}_{k}}=\lim _{|z| \rightarrow \infty} \exp \left(\frac{1}{2 \pi} \int_{0}^{2 \pi} \ln \frac{|\widetilde{\mathscr{F}}(z)|}{|\mathscr{F}(z)|} d \arg (z)\right) .
$$

Формула (2.5) и лемма 2 (см. Приложение) показывают, что последний предел равен $1+Q \sum_{k} a_{k}^{2} \mu_{k}$. Но из $\psi=\sum_{k} a_{k} u_{k}$ следует $\varphi=\sum_{k} \mu_{k} a_{k} u_{k}$, и потому

$$
\sum_{k=1}^{\infty} a_{k}^{2} \mu_{k}=\int_{\mathscr{O}} \psi(u) \varphi(u) d u=q .
$$

2) В статистической литературе формулу преобразования определителя Фредгольма при конечномерном возмущении оператора, частным случаем которой является (2.5), обычно возводят к работе [21]. Статистические приложения, полученные в этой работе, по-видимому, новы, но сама формула, полученная еще Бэйтменом [2], к тому времени уже была хорошо известна как в теории вычислительных методов, так и в спектральной теории операторов, причем в более общей ситуации (см., например, [11, гл. II, п. 4.6] и [1, п. 106]). 
Подставляя (2.6) в (2.4), получим (2.3). Теорема 1 доказана.

Рассмотрим теперь критический случай $\widehat{\alpha}=1 / q$.

Теорема 2. Пусть выполнено условие (2.1) и $\widehat{\alpha}=1 / q$. Если $\varphi \in$ $L_{2}(\mathscr{O})$, mо при $\varepsilon \rightarrow 0$

$$
\mathbf{P}\left\{\left\|\mathscr{X}_{\varphi, \hat{\alpha}}\right\|_{2} \leqslant \varepsilon\right\} \sim \frac{\sqrt{q}}{\|\varphi\|_{2}} \sqrt{\frac{2}{\pi}} \int_{0}^{\varepsilon^{2}} \frac{d}{d t} \mathbf{P}\left\{\|X\|_{2} \leqslant t\right\} \frac{d t}{\sqrt{\varepsilon^{2}-t^{2}}} .
$$

Д о к а з а т е л ь с т в о. Введем три функции распределения:

$$
\begin{aligned}
F(r) & =\mathbf{P}\left\{\sum_{k=1}^{\infty} \lambda_{k} \xi_{k}^{2} \leqslant r\right\}=\mathbf{P}\left\{\|X\|_{2} \leqslant \sqrt{r}\right\} ; \\
\widetilde{F}(r) & =\mathbf{P}\left\{\sum_{k=1}^{\infty} \tilde{\lambda}_{k} \xi_{k}^{2} \leqslant r\right\}=\mathbf{P}\left\{\left\|\mathscr{X}_{\varphi, \hat{\alpha}}\right\|_{2} \leqslant \sqrt{r}\right\} ; \\
F_{1}(r) & =\mathbf{P}\left\{\sum_{k=2}^{\infty} \lambda_{k} \xi_{k}^{2} \leqslant r\right\} .
\end{aligned}
$$

Аналогично предыдущей теореме,

$$
\widetilde{F}(r) \sim F_{1}(r)\left(\prod_{k=2}^{\infty} \frac{\widetilde{\mu}_{k-1}}{\mu_{k}}\right)^{1 / 2} \text { при } r \rightarrow 0 .
$$

Теорема Йенсена дает

$$
\prod_{k=2}^{\infty} \frac{\mu_{k}}{\widetilde{\mu}_{k-1}}=\lim _{|z| \rightarrow \infty} \exp \left(\frac{1}{2 \pi} \int_{0}^{2 \pi} \ln \left|\left(1-\frac{z}{\mu_{1}}\right) \frac{\widetilde{F}(z)}{\mathscr{F}(z)}\right| d \arg (z)\right) .
$$

Условие $\widehat{\alpha}=1 / q$ влечет $Q=-1 / q$, и выражение под знаком модуля можно переписать с учетом (2.5) и (2.7):

$$
\left(1-\frac{z}{\mu_{1}}\right)\left(1+Q \sum_{k=1}^{\infty} \frac{a_{k}^{2} \mu_{k}}{1-\mu_{k} / z}\right)=\frac{1 / \mu_{1}-1 / z}{q} \sum_{k=1}^{\infty} \frac{a_{k}^{2} \mu_{k}^{2}}{1-\mu_{k} / z}
$$

По лемме 2 (см. Приложение) предел в (2.9) равен

$$
\left(\mu_{1} q\right)^{-1} \sum_{k=1}^{\infty} a_{k}^{2} \mu_{k}^{2}=\left(\mu_{1} q\right)^{-1}\|\varphi\|_{2}^{2}
$$

откуда

$$
\widetilde{F}(r) \sim F_{1}(r) \frac{\sqrt{q \mu_{1}}}{\|\varphi\|_{2}} .
$$

Далее, очевидно, $F(r)=\left(F_{1} * f\right)(r)$, где

$$
f(x)=\frac{d}{d x} \mathbf{P}\left\{\lambda_{1} \xi^{2} \leqslant x\right\}=\frac{\exp \left(-x /\left(2 \lambda_{1}\right)\right)}{\sqrt{2 \pi \lambda_{1} x}} .
$$


C помощью преобразования Лапласа получаем решение этого сверточного уравнения:

$$
F_{1}(r)=\sqrt{\frac{2 \lambda_{1}}{\pi}} \exp \left(-\frac{r}{2 \lambda_{1}}\right) \int_{0}^{r}\left(F(x) \exp \left(\frac{x}{2 \lambda_{1}}\right)\right)^{\prime} \frac{d x}{\sqrt{r-x}} .
$$

Лемма 1. $F(x)=o\left(F^{\prime}(x)\right)$ при $x \rightarrow+0$.

Д о к а з а т е л ь с т в о. Заметим, что $F^{\prime}$ абсолютно непрерывна на $\mathbf{R}$ (если в сумме (2.2) есть хотя бы три ненулевых слагаемых). При этом $F^{\prime \prime}>0$ в правой полуокрестности нуля (это свойство непосредственно проверяется для трех слагаемых и, очевидно, сохраняется при добавлении нового слагаемого, причем радиус полуокрестности при этом не уменьшается; поэтому оно сохраняется и при переходе к бесконечной сумме). Таким образом, $F$ выпукла в правой полуокрестности нуля, и потому $F^{\prime}(x) \geqslant F(x) / x$ в этой полуокрестности. Лемма 1 доказана.

Продолжим доказательство теоремы. В силу леммы 1 из (2.11) получаем

$$
F_{1}(r) \sim \sqrt{\frac{2 \lambda_{1}}{\pi}} \int_{0}^{r} F^{\prime}(x) \frac{\exp \left(-(r-x) /\left(2 \lambda_{1}\right)\right) d x}{\sqrt{r-x}} \sim \sqrt{\frac{2 \lambda_{1}}{\pi}} \int_{0}^{r} F^{\prime}(x) \frac{d x}{\sqrt{r-x}} .
$$

Подставив это соотношение в (2.10) и сделав замену $r=\varepsilon^{2}$, получим (2.8). Теорема 2 доказана.

3 а м е ч а н и е. Формулу (2.10) можно (несколько вольно) интерпретировать так: при $\alpha \neq 1 / q$ последовательности $\mu_{k}$ и $\tilde{\mu}_{k}$ имеют одинаковые асимптотики; в условиях теоремы 2 , согласно п. 3 следствия 2 , одно из собственных чисел $\widetilde{\lambda}_{k}$ обращается в нуль, что порождает сбой в нумерации $\widetilde{\mu}_{k}$; изъятие из суммы $(2.2)$ лишнего слагаемого восстанавливает соответствие нумераций.

3. Разложение Карунена-Лоэва. Предположим теперь, что $n=$ $1, \mathscr{O}=(0, a)$ - интервал и ковариационная функция $G_{X}(t, s), t, s \in[0, a]$, является функцией Грина для самосопряженного оператора $L_{X}$ в пространстве $L_{2}(0, a)$, порождаемого дифференциальным выражением порядка $2 \ell$

$$
L_{X} u \equiv(-1)^{\ell} u^{(2 \ell)}+\left(p_{\ell-1} u^{(\ell-1)}\right)^{(\ell-1)}+\cdots+p_{0} u,
$$

с $2 \ell$ граничными условиями. Напомним, это означает, что функция $G_{X}$ при каждом $s \in(0, a)$ удовлетворяет уравнению $L_{X} G_{X}=\delta(t-s)$ в смысле обобщенных функций, а также граничным условиям. Не умаляя общности, можно считать, что $a=1$.

Обозначим $\mathscr{D}\left(L_{X}\right)$ образ интегрального оператора с ядром $G_{X}(t, s)$. Тогда легко видеть, что обратный оператор есть как раз $L_{X}$ с областью определения $\mathscr{D}\left(L_{X}\right)$. В частности, если $\varphi \in L_{2}(0,1)$, то $\psi \in \mathscr{D}\left(L_{X}\right)$ и $L_{X} \psi=\varphi$. 
Будем предполагать для простоты, что $p_{j} \in \mathscr{C}^{j}[0,1]$. Тогда $\mathscr{D}\left(L_{X}\right)$ совпадает с множеством функций, принадлежащих $W_{2}^{\ell}(0,1)$ и удовлетворяющих граничным условиям. С учетом (1.4) получаем, что ковариация преобразованного процесса $\mathscr{X}_{\varphi, \alpha}$ удовлетворяет уравнению

$$
L_{X} \mathscr{G}_{\varphi, \alpha}=\delta(t-s)+Q \varphi(t) \psi(s)
$$

(напомним, что $Q=q \alpha^{2}-2 \alpha$ ) и тем же граничным условиям.

Допустим, что нам известно КЛ-разложение (2.2) для исходного процесса. Тогда, очевидно, $u_{k}$ - собственные функции, а $\mu_{k}=\lambda_{k}^{-1}-$ собственные числа краевой задачи

$$
L_{X} u=\mu u, \quad u \in \mathscr{D}\left(L_{X}\right) .
$$

На практике собственные функции задачи (3.3) известны в аналитическом виде, только если при произвольном $\mu \in \mathbf{R}$ известна фундаментальная система решений уравнения $L_{X} v-\mu v=0$. Воспользуемся этим, чтобы дать алгоритм вычисления КЛ-разложения для процесса $\mathscr{X}_{\varphi, \alpha}$. Отметим, что в частном случае идея этого алгоритма применялась в [12] (см. далее пример 5).

Из (3.2) получаем краевую задачу для собственных функций интегрального оператора с ядром $\mathscr{G}_{\varphi, \alpha}(t, s)$ :

$$
L_{X} u=\mu u+\mu Q \varphi \int_{0}^{1} u(s) \psi(s) d s, \quad u \in \mathscr{D}\left(L_{X}\right) .
$$

Пусть $\mu$ - пока что неизвестный параметр. Методом вариации произвольных постоянных построим частное решение уравнения $L_{X} \eta-$ $\mu \eta=\varphi$. Тогда общее решение уравнения (3.4) записывается в виде

$$
u=c_{0} \eta+c_{1} v_{1}+c_{2} v_{2}+\cdots+c_{2 \ell} v_{2 \ell},
$$

где $v_{1}, \ldots, v_{2 \ell}$ - фундаментальная система решений уравнения $L_{X} v-$ $\mu v=0$. Подстановка в граничные условия дает $2 \ell$ уравнений для констант $c_{0}, c_{1}, \ldots, c_{2 \ell}$. Еще одно уравнение получаем, приравнивая коэффициенты при $\varphi$ в (3.4):

$$
\frac{c_{0}}{\mu Q}=c_{0} \int_{0}^{1} \eta(s) \psi(s) d s+c_{1} \int_{0}^{1} v_{1}(s) \psi(s) d s+\cdots+c_{2 \ell} \int_{0}^{1} v_{2 \ell}(s) \psi(s) d s .
$$

Собственные числа задачи (3.4) являются корнями определителя полученной однородной системы, а собственные функции - ее нетривиальными решениями.

Для иллюстрации алгоритма приведем несколько примеров.

П р и м е р 1. Пусть $X=W$ - винеровский процесс, $\varphi \equiv 1$. Тогда

$$
\psi(s)=\int_{0}^{1} \min (t, s) d s=\frac{2 t-t^{2}}{2}, \quad q=\int_{0}^{1} \frac{2 t-t^{2}}{2} d t=\frac{1}{3} .
$$


Соотношение (3.4) принимает вид

$$
-u^{\prime \prime}=\mu u+\frac{\mu Q}{2} \int_{0}^{1} u(s)\left(2 s-s^{2}\right) d s, \quad u(0)=u^{\prime}(1)=0 .
$$

Общее решение этого уравнения имеет вид

$$
u(t)=c_{0}+c_{1} \cos (\omega t)+c_{2} \sin (\omega t), \quad \omega=\mu^{1 / 2} .
$$

Подставляя его в граничные условия и в (3.5), выводим уравнение для собственных чисел:

$$
Q \sin (\omega)=\cos (\omega)\left(Q \omega+\left(1+\frac{Q}{3}\right) \omega^{3}\right)
$$

При $Q=0$ мы получаем $\cos (\omega)=0$, как и должно быть, поскольку в этом случае мы имеем обычный винеровский процесс. Отметим еще, что $1+Q / 3=(1-\alpha / 3)^{2} \geqslant 0$. При $\alpha=3$ уравнение сводится к $\operatorname{tg}(\omega)=\omega$.

Если $\omega_{k}$ - положительные корни уравнения (3.7), занумерованные в порядке возрастания, то в КЛ-разложении для процесса $\mathscr{W}_{\mathbf{1}, \alpha}$ имеем

$$
\tilde{\lambda}_{k}=\omega_{k}^{-2}, \quad \widetilde{u}_{k}(t)=\gamma_{k}\left(\cos \left(\omega_{k} t\right)-1+\operatorname{tg}\left(\omega_{k}\right) \sin \left(\omega_{k} t\right)\right),
$$

где $\gamma_{k}$ - нормирующие константы.

П р и м е р 2. Пусть $X=B-$ броуновский мост, $\varphi \equiv 1$. Тогда $\psi(t)=\left(t-t^{2}\right) / 2, q=\frac{1}{12}$. Соотношение (3.4) принимает вид

$$
-u^{\prime \prime}=\mu u+\frac{\mu Q}{2} \int_{0}^{1} u(s)\left(s-s^{2}\right) d s, \quad u(0)=u(1)=0 .
$$

Общее решение этого уравнения дается формулой (3.6). Подставляя его в граничные условия и в (3.5), выводим уравнение для собственных чисел:

$$
\left[\begin{array}{rl}
\sin (\tau) & =0 \\
Q \sin (\tau) & =\cos (\tau)\left(Q \tau+(4+Q / 3) \tau^{3}\right), \quad \tau=\frac{\omega}{2}
\end{array}\right.
$$

При $Q=0$ два уравнения в (3.9), как и следовало ожидать, объединяются в $\sin (\omega)=0$. Отметим еще, что $4+Q / 3=(2-\alpha / 6)^{2} \geqslant 0$. При $\alpha=12$ второе уравнение сводится к $\operatorname{tg}(\tau)=\tau$. Этот случай рассмотрен в $[6$, теорема 1.2].

Любопытным свойством обладает последовательность

$$
Q_{k}=-\frac{12}{1+3 /(k \pi)^{2}} \searrow-12
$$

(каждому $Q_{k}$ соответствуют два значения $\alpha$ ): при $Q=Q_{k} k$-е корни первой и второй серий в (3.9) совпадают. Таким образом, при $Q=Q_{1}$ 
задача (3.8) имеет кратное наименьшее собственное число - эффект, невозможный для обычной задачи Штурма-Лиувилля.

Если $\tau_{k}$ - положительные корни второй серии в (3.9), занумерованные в порядке возрастания, то в КЛ-разложении для процесса $\mathscr{B}_{\mathbf{1}, \alpha}$ имеем:

$$
\begin{array}{ll}
\widetilde{\lambda}_{k}=(2 k \pi)^{-2}, & \widetilde{u}_{k}(t)=\sqrt{2} \sin (2 \pi k t) \\
\widetilde{\widetilde{\lambda}}_{k}=\left(2 \tau_{k}\right)^{-2}, & \widetilde{\widetilde{u}}_{k}(t)=\gamma_{k}\left(\cos \left(2 \tau_{k} t\right)-1+\operatorname{tg}\left(\tau_{k}\right) \sin \left(2 \tau_{k} t\right)\right),
\end{array}
$$

где $\gamma_{k}$ - нормирующие константы.

П р и ме р 3. Пусть $X=B, \varphi(t)=t(1-t)$. Тогда $\psi(t)=$ $\left(t-2 t^{3}+t^{4}\right) / 12, q=\frac{17}{5040}$. Соотношение (3.4) принимает вид

$$
-u^{\prime \prime}=\mu u+\frac{\mu Q}{12} t(1-t) \int_{0}^{1} u(s)\left(s-2 s^{3}+s^{4}\right) d s, \quad u(0)=u(1)=0 .
$$

Общее решение этого уравнения дается формулой

$$
u(t)=c_{0}\left(t-t^{2}+\frac{2}{\omega^{2}}\right)+c_{1} \cos (\omega t)+c_{2} \sin (\omega t), \quad \omega=\mu^{1 / 2} .
$$

Подставляя его в граничные условия и в (3.5), выводим уравнение для собственных чисел:

$$
\left[\begin{array}{rl}
\sin (\tau) & =0 \\
Q \sin (\tau) & =\cos (\tau)\left(Q \tau+\frac{Q \tau^{3}}{3}+\frac{2 Q \tau^{5}}{15}+16(1+Q q) \tau^{7}\right), \quad \tau=\frac{\omega}{2}
\end{array}\right.
$$

При $Q=0$ два уравнения в $(3.10)$ объединяются в $\sin (\omega)=0$. Отметим, что $1+Q q=(1-q \alpha)^{2} \geqslant 0$.

Если $\tau_{k}$ - положительные корни второй серии в (3.10), занумерованные в порядке возрастания, то в КЛ-разложении для процесса $\mathscr{B}_{\varphi, \alpha}$ имеем

$$
\begin{array}{ll}
\widetilde{\lambda}_{k}=(2 k \pi)^{-2}, & \widetilde{u}_{k}(t)=\sqrt{2} \sin (2 \pi k t) ; \\
\widetilde{\widetilde{\lambda}}_{k}=\left(2 \tau_{k}\right)^{-2}, & \widetilde{\widetilde{u}}_{k}(t)=\gamma_{k}\left(\cos \left(2 \tau_{k} t\right)-1+2 \tau_{k}^{2}\left(t^{2}-t\right)+\operatorname{tg}\left(\tau_{k}\right) \sin \left(2 \tau_{k} t\right)\right),
\end{array}
$$

где $\gamma_{k}$ - нормирующие константы.

П р и м е р 4. Пусть

$$
X(t)=\bar{W}_{1}(t)=\int_{0}^{t}\left(W(s)-\int_{0}^{1} W(u) d u\right) d s
$$

- проинтегрированный центрированный винеровский процесс. Ковариация $G_{\bar{W}_{1}}$ (см., например, [10] и $[18$, предложение 5.4]) есть функция Грина для оператора $L_{\bar{W}_{1}}=L_{B}^{2}$. 
Если $\varphi \equiv 1$, то $\psi(t)=\left(t-2 t^{3}+t^{4}\right) / 24, q=\frac{1}{120}$ и соотношение $(3.4)$ принимает вид

$u^{\mathrm{IV}}=\mu u+\frac{\mu Q}{24} \int_{0}^{1} u(s)\left(s-2 s^{3}+s^{4}\right) d s, \quad u(0)=u(1)=u^{\prime \prime}(0)=u^{\prime \prime}(1)=0$.

Общее решение этого уравнения дается формулой

$$
u(t)=c_{0}+c_{1} \cos (\omega t)+c_{2} \sin (\omega t)+c_{3} \operatorname{ch}(\omega t)+c_{4} \operatorname{sh}(\omega t), \quad \omega=\mu^{1 / 4} .
$$

Подставляя его в граничные условия и в (3.5), выводим уравнение для собственных чисел:

$$
\left[\begin{array}{rl}
\sin (\tau) & =0 \\
Q(\sin (\tau)+\cos (\tau) \operatorname{th}(\tau)) & =\cos (\tau)\left(2 Q \tau+\left(32+\frac{4 Q}{15}\right) \tau^{5}\right), \quad \tau=\frac{\omega}{2}
\end{array}\right.
$$

При $Q=0$ два уравнения в $(3.11)$ объединяются в $\sin (\omega)=0$. Отметим, что $32+4 Q / 15=32(1-q \alpha)^{2} \geqslant 0$.

Если $\tau_{k}$ - положительные корни второй серии в (3.11), занумерованные в порядке возрастания, то в КЛ-разложении для процесса $\mathscr{X}_{\mathbf{1}, \alpha}$ имеем

$$
\begin{aligned}
\tilde{\lambda}_{k}=(2 k \pi)^{-4}, \quad \widetilde{u}_{k}(t) & =\sqrt{2} \sin (2 \pi k t) ; \\
\widetilde{\widetilde{\lambda}}_{k}=\left(2 \tau_{k}\right)^{-4}, \quad \widetilde{\widetilde{u}}_{k}(t)=\gamma_{k}( & \cos \left(2 \tau_{k} t\right)+\operatorname{ch}\left(2 \tau_{k} t\right)-2 \\
& \left.\quad+\operatorname{tg}\left(\tau_{k}\right) \sin \left(2 \tau_{k} t\right)-\operatorname{th}\left(\tau_{k}\right) \operatorname{sh}\left(2 \tau_{k} t\right)\right),
\end{aligned}
$$

где $\gamma_{k}$ - нормирующие константы.

Следует отметить, что в примерах $2-4$ собственные числа из первой серии в (3.9)-(3.11) не зависят от $Q$. Это связано с тем, что соответствующие собственные функции $\sin (2 \pi k t), k \in \mathbf{N}$, ортогональны $\psi$ в $L_{2}(0,1)$ и последний член в уравнении (3.4) обращается в нуль.

П р и м е р 5 . Пусть $X=B$ и

$\varphi(t)=\frac{1}{\phi\left(\boldsymbol{\Phi}^{-1}(t)\right)}, \quad$ где $\quad \phi(t)=\frac{1}{\sqrt{2 \pi}} \exp \left(-\frac{t^{2}}{2}\right), \quad \boldsymbol{\Phi}(x)=\int_{-\infty}^{x} \phi(t) d t$.

В этом важном для статистики примере (см. [12], [21]), очевидно, $\varphi \notin$ $L_{2}(0,1)$. Однако прямая выкладка дает $\psi=\phi\left(\boldsymbol{\Phi}^{-1}\right)=1 / \varphi, q=1$, и потому применимы все утверждения п. 1.

Далее, соотношение (3.4) принимает вид

$$
-u^{\prime \prime}=\mu u+\frac{\mu Q}{\phi\left(\boldsymbol{\Phi}^{-1}(t)\right)} \int_{0}^{1} u(s) \phi\left(\mathbf{\Phi}^{-1}(s)\right) d s, \quad u(0)=u(1)=0 .
$$

Общее решение этого уравнения дается формулой

$$
u(t)=c_{0} \int_{1 / 2}^{t} \frac{\sin (\omega(\tau-t)) d \tau}{\omega \phi\left(\boldsymbol{\Phi}^{-1}(\tau)\right)}+c_{1} \cos (\omega t)+c_{2} \sin (\omega t), \quad \omega=\mu^{1 / 2}
$$


Подставляя его в граничные условия и в (3.5), после некоторых преобразований получим уравнение для собственных чисел ${ }^{3)}$ :

$$
\operatorname{det}\left[\begin{array}{ccc}
0 & \int_{0}^{1} \boldsymbol{\Phi}^{-1}(\tau) \cos (\omega \tau) d \tau & -\frac{1}{\omega^{2} Q}+\int_{0}^{1} \int_{0}^{\tau} \boldsymbol{\Phi}^{-1}(\tau) \boldsymbol{\Phi}^{-1}(t) \frac{\sin (\omega(t-\tau))}{\omega} d t d \tau \\
\sin (\omega) & 0 & -\frac{1}{\omega} \int_{0}^{1} \boldsymbol{\Phi}^{-1}(\tau) \cos (\omega \tau) d \tau \\
\cos (\omega) & 1 & \frac{1}{\omega} \int_{0}^{1} \boldsymbol{\Phi}^{-1}(\tau) \sin (\omega \tau) d \tau
\end{array}\right]=0
$$

При $Q=0$ предельный переход дает, естественно, $\sin (\omega)=0$. Отметим, что здесь также половина из собственных функций исходного процесса, а именно, $\sin (2 \pi k t), k \in \mathbf{N}$, ортогональны $\psi$ в $L_{2}(0,1)$ и потому не меняются при изменении $Q$.

Опишем еще один случай, когда КЛ-разложение для преобразованного процесса строится элементарно. Пусть $\varphi=u_{m}$ - собственная функция ковариации $G_{X}$. Тогда $\psi=\lambda_{m} u_{m}$, где $\lambda_{m}$ - соответствующее собственное число. Поэтому все собственные функции $u_{k}, k \neq m$, ортогональны $\psi$, и, следовательно,

$$
\begin{array}{ll}
\tilde{\lambda}_{k}=\lambda_{k}, & \widetilde{u}_{k}=u_{k}, \quad k \neq m, \\
\widetilde{\lambda}_{m}=\lambda_{m}(1-q \alpha)^{2}, & \widetilde{u}_{m}=u_{m} .
\end{array}
$$

Установим теперь формулу, упрощающую (2.8) для процессов рассматриваемого класса.

Теорема 3. Пусть ковариачия $G_{X}$ является функцией Грина для оператора вида (3.1), $\widehat{\alpha}=1 / q$. Если $\varphi \in L_{2}(0,1)$, то при $\varepsilon \rightarrow 0$

$$
\mathbf{P}\left\{\left\|\mathscr{X}_{\varphi, \hat{\alpha}}\right\| \leqslant \varepsilon\right\} \sim \frac{\sqrt{q}}{\|\varphi\|_{2}}\left(2 \ell \sin \left(\frac{\pi}{2 \ell}\right) \varepsilon^{2}\right)^{-\ell /(2 \ell-1)} \cdot \mathbf{P}\{\|X\| \leqslant \varepsilon\} .
$$

Д о к а з а т е л ь с т в о. Как показано в [17, теорема 1.2] (случай оператора $L_{X}$ с разделенными граничными условиями был рассмотрен ранее в $[18, \S 7])$, для процесса $X$ справедливо соотношение

$$
F(r)=\mathbf{P}\{\|X\| \leqslant \sqrt{r}\} \sim \mathscr{C} r^{\beta} \exp \left(-\mathfrak{D} r^{-d}\right), \quad r \rightarrow 0,
$$

где $d=(2 \ell-1)^{-1}, \mathfrak{D}=(2 d)^{-1}\left(2 \ell \sin \left(\pi(2 \ell)^{-1}\right)\right)^{-d-1}$ (значения констант $\mathscr{C}$ и $\beta$ для нас сейчас несущественны).

Поведение плотности распределения $F^{\prime}(r)$ при малых $r$ изучалось в $[15$, теорема 3$]$ при весьма общих предположениях. В наших условиях (см. в связи с этим доказательство теоремы 6.2 [18]) этот результат [15] можно записать так:

$$
F^{\prime}(r) \sim \mathscr{C} \mathfrak{D} d r^{\beta-d-1} \exp \left(-\mathfrak{D} r^{-d}\right), \quad r \rightarrow 0,
$$

\footnotetext{
3) В [12] это уравнение записано в ином, эквивалентном, виде.
} 
что означает просто дифференцируемость асимптотики (3.13) по $r$.

Подставляя (3.14) и (2.12) в (2.10), получим

$$
\widetilde{F}(r) \sim \frac{\mathscr{C} \mathfrak{D} d}{\|\varphi\|_{2}} \sqrt{\frac{2 q}{\pi}} \int_{0}^{r} \frac{x^{\beta-d-1}}{\sqrt{r-x}} \exp \left(-\mathfrak{D} x^{-d}\right) d x
$$

Замена переменной $x=r(1-y)$ дает

$$
\begin{aligned}
\widetilde{F}(r) \sim & \frac{\mathscr{C} \mathfrak{D} d}{\|\varphi\|_{2}} \sqrt{\frac{2 q}{\pi}} r^{\beta-d-1 / 2} \exp \left(-\mathfrak{D} r^{-d}\right) \\
& \times \int_{0}^{1} \frac{(1-y)^{\beta-d-1}}{\sqrt{y}} \exp \left(-\frac{\mathfrak{D}}{r^{d}}\left((1-y)^{-d}-1\right)\right) d y .
\end{aligned}
$$

Легко видеть, что при $y \geqslant r^{d / 2}$ подынтегральное выражение экспоненциально мало. Поэтому можно интегрировать только по промежутку $\left[0, r^{d / 2}\right]$, на котором $(1-y)^{\beta-d-1} \sim 1$ и $(1-y)^{-d}-1 \sim y d$.

Сделав еще замену $y=r^{d} z$, получим

$$
\begin{aligned}
\widetilde{F}(r) & \sim \frac{\mathscr{C} \mathfrak{D} d}{\|\varphi\|_{2}} \sqrt{\frac{2 q}{\pi}} r^{\beta-(d+1) / 2} \exp \left(-\mathfrak{D} r^{-d}\right) \int_{0}^{r^{-d / 2}} \frac{1}{\sqrt{z}} \exp (-d \mathfrak{D} z) d z \\
& \sim \frac{\mathscr{C}}{\|\varphi\|_{2}} \sqrt{2 q \mathfrak{D} d} r^{\beta-(d+1) / 2} \exp \left(-\mathfrak{D} r^{-d}\right) \sim \frac{\sqrt{2 q \mathfrak{D} d}}{\|\varphi\|_{2}} r^{-(d+1) / 2} F(r),
\end{aligned}
$$

что дает (3.12). Теорема 3 доказана.

В примерах, рассмотренных выше, асимптотика малых уклонений для исходных процессов хорошо известна (процессы $W$ и $B$ являются классическими, процесс $\bar{W}_{1}$ разобран в [3]). Применяя теоремы 1 и 3 , получаем следующее предложение.

Предложение 1. При $\varepsilon \rightarrow 0$ имеем:

$$
\begin{aligned}
& \mathbf{P}\left\{\left\|\mathscr{W}_{\mathbf{1}, \alpha}\right\| \leqslant \varepsilon\right\} \sim\left[\begin{array}{ll}
\frac{4 \varepsilon}{\sqrt{\pi}|1-\alpha / 3|} \exp \left(-\frac{1}{8} \varepsilon^{-2}\right), & \alpha \neq 3 \\
\frac{2 \varepsilon^{-1}}{\sqrt{3 \pi}} \exp \left(-\frac{1}{8} \varepsilon^{-2}\right), & \alpha=3
\end{array}\right. \\
& \mathbf{P}\left\{\left\|\mathscr{B}_{1, \alpha}\right\| \leqslant \varepsilon\right\} \sim\left[\begin{array}{ll}
\frac{2 \sqrt{2}}{\sqrt{\pi}|1-\alpha / 12|} \exp \left(-\frac{1}{8} \varepsilon^{-2}\right), & \alpha \neq 12, \\
\frac{\varepsilon^{-2}}{\sqrt{6 \pi}} \exp \left(-\frac{1}{8} \varepsilon^{-2}\right), & \alpha=12 ;
\end{array}\right. \\
& \mathbf{P}\left\{\left\|\mathscr{B}_{\varphi, \alpha}\right\| \leqslant \varepsilon\right\} \sim\left[\begin{array}{ll}
\frac{2 \sqrt{2}}{\sqrt{\pi}|1-17 \alpha / 5040|} \exp \left(-\frac{1}{8} \varepsilon^{-2}\right), & \alpha \neq \frac{5040}{17} \\
\frac{\sqrt{17} \varepsilon^{-2}}{2 \sqrt{21 \pi}} \exp \left(-\frac{1}{8} \varepsilon^{-2}\right), & \alpha=\frac{5040}{17}
\end{array}\right. \\
& (\text { здесь } \varphi(t)=t(1-t)) \text {; }
\end{aligned}
$$




$$
\begin{aligned}
& \mathbf{P}\left\{\left\|\mathscr{X}_{\mathbf{1}, \alpha}\right\| \leqslant \varepsilon\right\} \sim\left[\begin{array}{ll}
\frac{4 \sqrt{2} \varepsilon^{-1 / 3}}{\sqrt{3 \pi}|1-\alpha / 120|} \exp \left(-\frac{3}{8} \varepsilon^{-2 / 3}\right), & \alpha \neq 120 \\
\frac{\varepsilon^{-5 / 3}}{3 \sqrt{5 \pi}} \exp \left(-\frac{3}{8} \varepsilon^{-2 / 3}\right), & \alpha=120
\end{array}\right. \\
& \text { (здесь } \left.X(t)=\bar{W}_{1}(t)\right) \text {; } \\
& \mathbf{P}\left\{\left\|\mathscr{B}_{\varphi, \alpha}\right\| \leqslant \varepsilon\right\} \sim \frac{2 \sqrt{2}}{\sqrt{\pi}|1-\alpha|} \exp \left(-\frac{1}{8} \varepsilon^{-2}\right), \quad \alpha \neq 1 \\
& \left(\text { здесь } \varphi(t)=\frac{1}{\phi\left(\boldsymbol{\Phi}^{-1}(t)\right)}\right) \text {. }
\end{aligned}
$$

3 а м е ч а н и е. В примере 5 при $\alpha=1$ теорема 2 (и, следовательно, теорема 3) не применима.

4. Некоторые обобщения. Конструкция (1.3) может быть распространена на некоторый специальный класс обобщенных функций $\varphi$.

Пусть $\varphi \in D^{\prime}(\mathscr{O})$ такова, что $q \equiv \mathbf{E}|\langle\varphi, X\rangle|^{2}<\infty$. Определим семейство гауссовских функций формулой, аналогичной (1.3):

$$
\mathscr{X}_{\varphi, \alpha}(x)=X(x)-\alpha \psi(x)\langle\varphi, X\rangle, \quad x \in \overline{\mathscr{O}}
$$

где $\psi(x)=\mathbf{E} X(x)\langle\varphi, X\rangle$.

3 а м е ч а н и е. В терминах теории операторов условие на $\varphi$ означает, что $\varphi \in\left(\operatorname{Im}\left(\mathfrak{G}^{1 / 2}\right)\right)^{\prime}$. Соответственно, $\psi \in \operatorname{Im}\left(\mathfrak{G}^{1 / 2}\right)$ и $q=\langle\varphi, \psi\rangle$. В теории случайных процессов $\operatorname{Im}\left(\mathfrak{G}^{1 / 2}\right)$ именуется ядром распределения процесса $X$, а $\varphi$ - линейным измеримым функционалом от процесса $X$, ассоциированным с $\psi$ (см. $[14, \S 9])$. Отметим, что формула (4.1) дает общий вид одномерного линейного возмущения гауссовских случайных процессов.

Для процессов (4.1) выполнены основная лемма и следствие 1. Вместо следствия 2 имеет место следующий его аналог.

Следствие $2^{\prime}$. При $\widehat{\alpha}=1 / q$ прочесс $\mathscr{X}_{\varphi, \hat{\alpha}}(x)$ и случайная величина $\langle\varphi, X\rangle$ независимы. Кроме того, $\left\langle\varphi, \mathscr{X}_{\varphi, \hat{\alpha}}\right\rangle=0$ п.н.

Далее, при выполнении условия (2.1) справедлива теорема 1. Если же ковариация $G_{X}(t, s)$ удовлетворяет условиям, описанным в п. 3 , то проходит и алгоритм построения КЛ-разложения для процесса $\mathscr{X}_{\varphi, \alpha}$.

Приведем несколько примеров.

П р и м е р 6. Пусть $X=W, \varphi(t)=\delta(t-1)$. Тогда $\psi(t)=$ $G_{W}(t, 1)=t, q=G_{W}(1,1)=1$ и

$$
\mathscr{G}_{\varphi, \alpha}(t, s)=\min \{t, s\}+Q t s \quad\left(Q=\alpha^{2}-2 \alpha\right) .
$$

Таким образом, при $\alpha \in] 0,2\left[\right.$ процесс $\mathscr{W}_{\varphi, \alpha}$ совпадает по распределению с броуновским мостом от нуля до нуля длины $-1 / Q$ (см. $[5,4.4 .20])$. 
Теорема 1 дает для $\alpha \neq 1$

$$
\mathbf{P}\left\{\left\|\mathscr{W}_{\varphi, \alpha}\right\| \leqslant \varepsilon\right\} \sim \frac{4 \varepsilon}{\sqrt{\pi}|1-\alpha|} \exp \left(-\frac{1}{8} \varepsilon^{-2}\right), \quad \varepsilon \rightarrow 0,
$$

что совпадает с частными случаями $[16$, предложение 1.9$]$ и $[19$, теорема 4.1].

3 а м е ч а н и е. При $\alpha=1$ процесс $\mathscr{W}_{\varphi, \alpha}$ совпадает по распределению со стандартным броуновским мостом. Легко видеть, что бесконечное произведение в (2.9) при этом расходится, и теорема 2 места не имеет (как и в дальнейших примерах).

П р и м е р 7 . Рассмотрим проинтегрированный винеровский процесс

$$
X(t)=W_{1}^{[0]}(t)=\int_{0}^{t} W(s) d s .
$$

Если $\varphi(t)=\delta^{\prime}(t-1)$, то $\psi(t)=-\left(G_{W_{1}}\right)_{s}(t, 1)=-t^{2} / 2, q=$ $\left(G_{W_{1}}\right)_{s t}(1,1)=1$ и

$$
\mathscr{G}_{\varphi, \alpha}(t, s)=G_{W_{1}}(t, s)+Q \frac{t^{2} s^{2}}{4} \quad\left(Q=\alpha^{2}-2 \alpha\right) .
$$

Таким образом, процесс $\mathscr{X}_{\varphi, \alpha}$ совпадает по распределению с проинтегрированным процессом из примера 6. Утверждение теоремы 1 соответствует случаю $m=1$ в [16, предложение 1.9]. Малые уклонения в случае $\alpha=1$ (проинтегрированный броуновский мост) рассмотрены в [16, предложение 1.6].

Пусть теперь $\varphi(t)=\delta(t-1)$. Тогда $\psi(t)=G_{W_{1}}(t, 1)=t^{2} / 2-t^{3} / 6$ и $q=G_{W_{1}}(1,1)=\frac{1}{3}$. Теорема 1 дает для $\alpha \neq 3$

$$
\mathbf{P}\left\{\left\|\mathscr{X}_{\varphi, \alpha}\right\| \leqslant \varepsilon\right\} \sim \frac{8 \sqrt{6} \varepsilon^{1 / 3}}{\sqrt{\pi}|3-\alpha|} \exp \left(-\frac{3}{8} \varepsilon^{-2 / 3}\right), \quad \varepsilon \rightarrow 0 .
$$

При $\alpha=3$ можно прямым вычислением проверить, что $\mathscr{G}_{\varphi, \alpha}(t, s)$ есть функция Грина краевой задачи

$$
u^{\mathrm{IV}}=\mu u ; \quad u(0)=u^{\prime}(0)=u(1)=u^{\prime \prime}(1)=0 .
$$

Применяя теорему 1.4 из работы [16], получаем

$$
\mathbf{P}\left\{\left\|\mathscr{X}_{\varphi, \alpha}\right\| \leqslant \varepsilon\right\} \sim \frac{4 \sqrt{2} \varepsilon^{-2 / 3}}{3 \sqrt{\pi}} \exp \left(-\frac{3}{8} \varepsilon^{-2 / 3}\right), \quad \varepsilon \rightarrow 0 .
$$

П р и м е р 8. Рассмотрим еще процесс Слепяна [20] - стационарный гауссовский процесс с нулевым средним и ковариацией $G_{S}(t, s)=$ $1-|t-s|, t, s \in[0,1]$. Пусть $\varphi(t)=\delta(t)+\delta(t-1)$. Тогда $\psi \equiv 1, q=2$ и

$$
\mathscr{G}_{\varphi, \alpha}(t, s)=1+Q-|t-s| \quad\left(Q=2 \alpha^{2}-2 \alpha\right) .
$$


Таким образом, процесс $\mathscr{S}_{\varphi, \alpha}$ совпадает по распределению с обобщенным процессом Слепяна $S^{(c)}, c=1+Q$ (см. [9] и $\left.[17, \S 2]\right)$. Отметим, что при $Q \geqslant 0$ справедливо также равенство

$$
S^{(c)}(t) \stackrel{d}{=} W(t+c)-W(t), \quad 0 \leqslant t \leqslant 1 .
$$

Утверждение теоремы 1 соответствует [17, теорема 2.1, п. 2]. Малые уклонения в случае $\alpha=1 / 2$ (т.е. $c=1 / 2$ ) рассмотрены в [17, теорема 2.1 , п. 1].

П р и м е р 9. Аналогично, несложно показать, что к теореме 1 сводится утверждение [16, предложение 1.9] при произвольном $m \in \mathbf{N}$, а также $[17$, теорема 2.2 , п. 2] и некоторые утверждения из [19]. Таким образом, эта теорема дает единый подход к многим формулам, полученным ранее.

Можно также рассматривать многопараметрические аналоги преобразования (1.3). Мы ограничимся простейшим случаем.

Пусть функции $\varphi_{1}, \varphi_{2} \in L_{1, \text { loc }}(\mathscr{O})$ удовлетворяют условию (1.2) и условию «ортогональности»)

$$
\int_{\mathscr{O}} \int_{\mathscr{O}} G_{X}(u, v) \varphi_{1}(u) \varphi_{2}(v) d u d v=0 .
$$

Рассмотрим семейство гауссовских функций

$$
\mathscr{X}_{\boldsymbol{\varphi}, \boldsymbol{\alpha}}(x)=X(x)-\alpha_{1} \psi_{1}(x) \int_{\mathscr{O}} \begin{gathered}
X(u) \varphi_{1}(u) d u-\alpha_{2} \psi_{2}(x) \int_{\mathscr{O}} X(u) \varphi_{2}(u) d u, \\
x \in \overline{\mathscr{O}},
\end{gathered}
$$

где $\psi_{k}=\mathfrak{G} \varphi_{k}, k=1,2$.

Легко видеть, что функция (4.2) имеет ковариацию

$$
\mathscr{G}_{\boldsymbol{\varphi}, \boldsymbol{\alpha}}(x, y)=G_{X}(x, y)+Q_{1} \psi_{1}(x) \psi_{1}(y)+Q_{2} \psi_{2}(x) \psi_{2}(y),
$$

где $Q_{k}=q_{k} \alpha_{k}^{2}-2 \alpha_{k}, q_{k}=\left\langle\varphi_{k}, \psi_{k}\right\rangle$. Поэтому, например, асимптотика $L_{2}$-малых уклонений функции $\mathscr{X}_{\boldsymbol{\varphi}, \boldsymbol{\alpha}}$ при $\alpha_{k} \neq 1 / q_{k}, k=1,2$, получается двукратным применением теоремы 1 . Так же получаются аналоги остальных утверждений.

\section{5. Приложение.}

Лемма 2. Рассмотрим две последовательности; $\mu_{k}>0 u b_{k} \geqslant 0$, $k \in \mathbf{N}$. Пусть $\sum_{k} \mu_{k}^{-1}<\infty u \sum_{k} b_{k}<\infty$. Тогда при $R \rightarrow \infty$

$$
\frac{1}{2 \pi} \int_{0}^{2 \pi} \ln \left|\gamma_{1}+\gamma_{2} \sum_{k=1}^{\infty} \frac{b_{k}}{1-\mu_{k} /(R \exp (i \theta))}\right| d \theta \longrightarrow \ln \left(\gamma_{1}+\gamma_{2} \sum_{k=1}^{\infty} b_{k}\right)
$$

для любых $\gamma_{1}, \gamma_{2} \in \mathbf{R}$ таких, что выражение под знаком логарифма в правой части положительно.

4) Для обобщенных функций $\varphi_{1}, \varphi_{2} \in\left(\operatorname{Im}\left(\mathfrak{G}^{1 / 2}\right)\right)^{\prime}$ это условие принимает вид $\left\langle\varphi_{1}, \mathfrak{G} \varphi_{2}\right\rangle=0$. 
Д о к а з а т е л ь с т в о. При любом фиксированном $\theta \in] 0,2 \pi[$ выражение $1-\mu_{k} /(R \exp (i \theta))$ отделено от нуля. По теореме Лебега можно перейти к пределу под знаком суммы. Таким образом, подынтегральное выражение в (5.1) сходится к $\ln \left(\gamma_{1}+\gamma_{2} \sum_{k} b_{k}\right)$ при $\left.\theta \in\right] 0,2 \pi[$, причем сходимость равномерная на любом отрезке.

Далее, выражение под знаком модуля имеет лишь простые нули и полюсы. Поэтому подынтегральное выражение в (5.1) имеет лишь логарифмические особенности, и потому несложно построить суммируемую мажоранту вида const $\cdot \ln |\sin (\theta)|$. Повторное применение теоремы Лебега заканчивает доказательство.

Я весьма признателен М.А. Лифшицу и Я.Ю. Никитину за стимулирующие обсуждения и библиографические указания, а также М. Ш. Бирману, указавшему мне на работу [2].

\section{СПИСОК ЛИТЕРАТУРЫ}

1. Ахиезер Н. И., Глазман И. М. Теория линейных операторов в гильбертовом пространстве. М.: Наука, 1966, 543 с.

2. Bateman $H$. A formula for the solving function of a certain integral equation of the second kind. - Messenger Math., 1908, v. 37, p. 179-187.

3. Beghin L., Nikitin Ya. Yu., Orsingher E. Exact small ball constants for some Gaussian processes under the $L_{2}$-norm. - Записки науч. сем. ПОМИ, 2003, т. 298, с. 5-21.

4. Бирман М. ШІ, Соломяк М. З. Спектральная теория самосопряженных операторов в гильбертовом пространстве. Л.: Изд-во ЛГУ, 1980, 264 с.

5. Бородин А.Н., Салминен П. Справочник по броуновскому движению. Факты и формулы. СПб.: Лань, 2000, 639 с.

6. Deheuvels P. A Karhunen-Loève expansion for a mean-centered Brownian bridge. Statist. Probab. Lett., 2007, v. 77, № 12, p. 1190-1200.

7. Dunker T., Lifshits M. A., Linde W. Small deviations probabilities of sums of independent variables. - Progr. Probab., 1998, v. 43, p. 59-74.

8. Gao F., Hannig J., Torcaso F. Comparison theorems for small deviations of random series. - Electron. J. Probab., 2003, v. 8, № 21, p. 1-17.

9. Gao F., Li W. V. Small ball probabilities for the Slepian Gaussian fields. - Trans. Amer. Math. Soc., 2007, v. 359, № 3, p. 1339-1350.

10. Henze N., Nikitin Ya. Yu. Watson-type goodness-of-fit tests based on the integrated empirical process. - Math. Methods Statist., 2002, v. 11, № 2, p. 183-202.

11. Канторович Л. В., Крылов В. И. Приближенные методы высшего анализа. М.-Л.: Физматгиз, 1962, 708 с.

12. Kac M., Kiefer J., Wolfowitz J. On tests of normality and other tests of goodness of fit based on the minimum distance methods. - Ann. Math. Statist., 1955, v. 26, № 2 , p. 189-211.

13. $L i \quad W$. $V$. Comparison results for the lower tail of Gaussian seminorms. - J. Theoret. Probab., 1992, v. 5, № 1, p. 1-31.

14. Лифиии М. А. Гауссовские случайные функции. Киев: ТВиМС, 1995.

15. Lifshits $M$. A. On the lower tail probabilities of some random series. - Ann. Probab., 1997 , v. 25 , № 1, p. 424-442.

16. Назаров А.И. О точной константе в асимптотике малых уклонений в $L_{2}$-норме некоторых гауссовских процессов. - Нелинейные задачи и теория функций. Под ред. Н. Н. У ральцевой. Новосибирск: Тамара Рожковская, с. 179-214. (Проблемы матем. анализа, в. 26.) 
17. Nazarov A. I. Exact $L_{2}$-small ball asymptotics of Gaussian processes and the spectrum of boundary value problems. - J. Theoret. Probab., 2008. DOI 10.1007/s10959-0080173-7.

18. Nazarov A.I., Nikitin Ya. Yu. Exact $L_{2}$-small ball behavior of integrated Gaussian processes and spectral asymptotics of boundary value problems. - Probab. Theory Related Fields, 2004, v. 129, № 4, p. 469-494.

19. Назаров А.И., Пусев Р. С. Точная асимптотика малых уклонений в $L_{2}$-норме с весом для некоторых гауссовских процессов. - Записки науч. сем. ПОМИ, 2009, т. 364 , c. $166-199$.

20. Slepian D. First passage time for a particular Gaussian process. - Ann. Math. Statist., 1961, v. 32, p. 610-612.

21. Sukhatme $S$. Fredholm determinant of a positive definite kernel of a special type and its application. - Ann. Math. Statist., 1972, v. 43, №6, p. 1914-1926.

22. Сытая Г.Н. О некоторых асимптотических представлениях гауссовской меры в гильбертовом пространстве. - Теория случайных процессов, 1974, т. 2, с. 93-104.

23. Титчмари E. Теория функций. М.: Наука, 1980, 463 с.

Поступила в редакцию 2.IV.2008 